\title{
Quantitative reconstruction of absorption and scattering coefficients in ultrasound-modulated optical tomography
}

\author{
Samuel Powell ${ }^{a}$ and Terence S. Leung ${ }^{a}$ \\ ${ }^{a}$ University College London, Department of Medical Physics and Bioengineering, Malet Place \\ Engineering Building, LONDON, WC1E 6EB.
}

\begin{abstract}
The simultaneous and/or quantitative recovery of optical absorption and scattering coefficients in ultrasoundmodulated optical tomography requires the use of a model-based inversion procedure. In this work we employ a linearised forward model as part of a non-linear image reconstruction process, recovering parameters with an error of less than $\pm 3 \%$ from simulated measurements with $1 \%$ Gaussian noise and initial conditions differing by $10 \%$ from the actual background.
\end{abstract}

Keywords: Ultrasound-modulated optical tomography, acousto-optics, image reconstruction, optical tomography, imaging

\section{INTRODUCTION}

Ultrasound modulated optical tomography (UOT) is a hybrid imaging technique which combines the spatial resolution of ultrasound fields and the optical contrast of near infra-red light in biological media. A principle goal of this developing technology is the reconstruction of clinically relevant quantitative images of the absorption and scattering coefficients in biological media, with a spatial resolution exceeding that of purely optical techniques such as diffuse optical tomography (DOT). ${ }^{1}$

Much effort has been expended in advancing the experimental technique in UOT. The problem of detecting the small ultrasound-modulated optical flux against the large unmodulated background has received significant attention. This problem is particularly challenging since the requisite use of a coherent source generates a spatially incoherent speckle pattern on the boundary of the domain. The flux of individual coherence areas must therefore be collected in parallel, ${ }^{2-4}$ or manipulated such that their contributions can be measured in summation..$^{5-8}$

Less attention has been paid to the fundamental problem that hybrid techniques such as UOT are only capable of producing quantitative images under some form of model-based reconstruction procedure. This was succinctly demonstrated by the images produced by Lev and $\mathrm{Sfez}^{9,10}$ where it was demonstrated that a UOT measurement is akin to a scaled sample of the optical absorption sensitivity function ${ }^{11}$ for a given domain. Bratchenia et al. previously developed a non-linear reconstruction algorithm capable of recovering the optical absorption coefficient from a UOT experiment. ${ }^{12}$ We previously demonstrated the recovery of the optical absorption coefficient in a linear inversion, and investigated the form of the UOT absorption sensitivity functions. ${ }^{13}$

The more subtle problem of non-uniqueness in the simultaneous recovery of both the optical absorption and scattering coefficients has not been tackled for coherent ultrasound-modulated optical tomography, though the theoretical basis has been tackled for an alternative incoherent formulation by Bal et al. (see 14,15 and related publications). The key idea is that the recovery of $2 N$ unknowns (the two coefficients) requires at least two sets of internal data. In this work we develop a non-linear reconstruction technique which employs multiple optical source-detector pairs to restore uniqueness and thus permit the simultaneous, quantitative recovery of both the optical absorption and scattering coefficients.

Further author information: (Send correspondence to S. Powell)

S. Powell: E-mail: s.powell@ucl.ac.uk, Telephone: +44 (0) 20726790273

Photons Plus Ultrasound: Imaging and Sensing 2014, edited by Alexander A. Oraevsky, Lihong V. Wang, Proc. of SPIE Vol. 8943, 89434X · C 2014 SPIE · CCC code: 1605-7422/14/\$18 · doi: 10.1117/12.2039770 


\section{THEORY}

We describe our reconstruction procedure in two parts. First, we define a forward model describing the generation and propagation of our modulated optical field. Second, we define an optimisation procedure to recover the pertinent coefficients from measurements of this field made on the boundary of the domain.

\subsection{Forward model}

A coherent optical field is modulated by a quasi-monochromatic acoustic field distribution. We take the modulated component of the optical fluence in the domain, $\phi_{a}(\mathbf{r})$, to be given by,

$$
\left[-\nabla \cdot D(\mathbf{r}) \nabla+\mu_{a}(\mathbf{r})\right] \phi_{a}(\mathbf{r})=q_{a}(\mathbf{r}), \quad \mathbf{r} \in \Omega,
$$

where $D(\mathbf{r})=1 /\left(3 \mu_{s}^{\prime}(\mathbf{r})\right)$ is the diffusion coefficient, $\mu_{a}(\mathbf{r})$ is the absorption coefficient, and $q_{a}(\mathbf{r})$ is the UOT 'virtual source'. This source term is given by,

$$
q_{a}(\mathbf{r})=\eta_{a}(\mathbf{r}) \phi_{0}(\mathbf{r})
$$

where $\eta_{a}(\mathbf{r})$ is the acousto-optic modulation efficiency, $\phi_{0}(\mathbf{r})$ is the solution to the standard diffusion approximation in DOT,

$$
\left[-\nabla \cdot D(\mathbf{r}) \nabla+\mu_{a}(\mathbf{r})\right] \phi_{0}(\mathbf{r})=q_{0}(\mathbf{r}), \quad \mathbf{r} \in \Omega,
$$

and $q_{0}(\mathbf{r})$ is an isotropic source of coherent light. A coherent collimated source is approximated by the placement of an isotropic source at a distance of $1 / \mu_{s}^{\prime}(\mathbf{r})$ below and normal to the surface upon which the collimated source is incident. Each diffusion equation is equipped with a Robin (impedance) style boundary condition, ${ }^{16}$

$$
\phi_{x}(\mathbf{r})+2 A \mathbf{n} \cdot D(\mathbf{r}) \nabla \phi_{x}(\mathbf{r})=0, \quad \mathbf{r} \in \partial \Omega,
$$

where $A$ relates to the index of refraction mismatch between the domain and that by which it is surrounded, $\mathbf{n}$ is the unit outward normal to the boundary, and $\phi_{x}(\mathbf{r})$ may be either $\phi_{0}(\mathbf{r})$ or $\phi_{a}(\mathbf{r})$.

Our measurement is that of the modulated flux across the boundary of the domain. Under the assumption of a matched index of refraction $(A=1)$ this is given by $y=\phi_{a}(\mathbf{r}) / 2, \mathbf{r} \in \partial \Omega$; this quantity may be measured directly or inferred from data collected by many of the detection mechanisms featured in the literature. In the case of a finite detection aperture the flux may be integrated over the area of the detector.

Our model was presented in different forms by both Allmaras and Bangerth, ${ }^{17}$ and Bratchenia et al. ${ }^{12}$ The former work provides a reasoned derivation based upon a path integral formulation, the nature of those paths then being formalised in the diffusion framework. This model can be also be derived from the time (lag) domain correlation diffusion approximation presented by by Sakadžić and Wang, ${ }^{18}$

$$
\left[-\nabla \cdot D(\mathbf{r}) \nabla+\mu_{a}(\mathbf{r})+\alpha(\mathbf{r})\left(1-\cos \left(\omega_{a} \tau\right)\right)\right] \phi(\mathbf{r}, \tau)=q_{0}(\mathbf{r}), \quad \mathbf{r} \in \Omega,
$$

where $\phi(\mathbf{r}, \tau)$ is the temporal field autocorrelation function, and $\alpha(\mathbf{r})$ is dependent upon the square of the acoustic pressure amplitude, and the optical and acoustic properties of the domain. By linearising this expression in the small parameter $\alpha$, and taking the Fourier transform of the resulting expressions at the acoustic frequency, we arrive at equations 1 to 3 where $\alpha(\mathbf{r})$ is subsumed into the modulation efficiency term $\eta_{a}(\mathbf{r})$ (this linearisation can be seen in a different setting in the work of Varma et al. ${ }^{19}$ ). We now see that our measurement in the prescribed model is the power-spectral density of the coherent flux emanating from the sample at the acoustic frequency.

For the case of continuous wave (CW) acoustic modulation with a focused field, we may manipulate $\alpha(\mathbf{r})$ (as given by Sakadžić and Wang ${ }^{18}$ ) to approximate the expected acousto-optic modulation efficiency. If we assume, in addition to the standard assumptions made in the diffusion approximation, that $k_{a} l_{\mathrm{tr}} \gg 1$, where $k_{a}$ is the acoustic wavenumber, and $l_{\mathrm{tr}}=1 /\left(\mu_{a}(\mathbf{r})+\mu_{s}^{\prime}(\mathbf{r})\right)$, we find $\eta_{a}(\mathbf{r}) \approx \beta(\mathbf{r}) P_{0}^{2}(\mathbf{r})$. The term $\beta(\mathbf{r})$ is principally dependent upon the wavelength of illumination and insonification, the refractive index of the domain, and the acousto-optic coefficient; in the prescribed conditions, the weak dependence upon the optical properties of the domain are neglected. 


\subsection{Reconstruction procedure}

In UOT we take a number of measurements $y_{m, c}$ indexed over $c$ different acoustic field distributions, and optical source/detector locations. Here, the continuous wave formulation implies the scanning of a focused acoustic field through the medium; though this could be extended to consider a time-gated measurement where the propagation of a pulse defines a temporo-spatial distribution. The use of multiple source/detector locations is required to resolve the non-uniqueness extant in the UOT problem. This approach is equivalent to the multiple-illumination technique in photo-acoustics, and we shall see its effect on the ill-posedness of the problem in section 4.

To perform a reconstruction in a least-squares sense, we define a regularised objective functional,

$$
\Psi(\mathbf{x})=\frac{1}{2} \sum_{c}\left|y_{m, c}-y_{c}(\mathbf{x})\right|^{2}+\lambda R(\mathbf{x})
$$

where $\mathbf{x}=\left[\mu_{a}(\mathbf{r}) \mu_{s}^{\prime}(\mathbf{r})\right]^{T}$ consists of the parameters we wish to recover, and $y_{c}(\mathbf{x})$ is the output of the forward model defined in section 2.1 with the optical and acoustical configuration indicated by index $c$. The practical inverse problem in UOT remains ill-posed irrespective of the use of multiple illumination profiles, this is fundamentally due to the diffusive nature of the light transport problem; but the degree of ill-posedness is also affected by how we choose to sample our domain with a choice of acoustic field distributions. To permit the solution of the problem in a meaningful way therefore requires us to introduce prior information regarding the expected structure of the solution. This task is achieved by the regularisation functional $R(\mathbf{x})$, the contribution of which is scaled by the hyper-parameter $\lambda$. A good solution, given our prior knowledge, is then given by finding

$$
\mathbf{x}_{g}=\underset{\mathbf{x}}{\operatorname{argmin}} \Psi(\mathbf{x})
$$

There are numerous methods by which this optimisation procedure may be performed. For the small scale two-dimensional problem presented in this work, we can choose a scheme which offers rapid convergence but might otherwise be impracticable due to memory requirements. Here, we employ the damped Gauss-Newton method, which is derived as follows.

1. The Newton update equation is sought by expanding the objective function in a second order Taylor series, taking the derivative, and equating to zero.

2. The second order Fréchet derivatives are replaced with an approximation based upon the first order derivatives, resulting in the Gauss-Newton method.

3. A scaling factor is introduced to the update equation which is found by a line-search of the un-approximated forward model.

The next estimate of the parameters is thus given by, ${ }^{20}$

$$
\mathbf{x}^{(k+1)}=\mathbf{x}^{(k)}+\zeta\left(y_{c}^{\prime *}\left(\mathbf{x}^{(k)}\right) y_{c}^{\prime}\left(\mathbf{x}^{(k)}\right)+\lambda R^{\prime \prime}\left(\mathbf{x}^{(k)}\right)\right)^{-1}\left(y_{c}^{\prime *}\left(\mathbf{x}^{(k)}\right)\left(y_{m, c}-y_{c}\left(\mathbf{x}^{(k)}\right)\right)+\lambda R^{\prime}\left(\mathbf{x}^{(k)}\right)\right),
$$

where $y_{c}^{\prime *}\left(\mathbf{x}^{(k)}\right)$ represents the conjugate of the first Frechét derivative of the forward model with respect to the parameters at iteration $k$, and $R^{\prime}\left(\mathbf{x}^{(k)}\right)$ and $R^{\prime \prime}\left(\mathbf{x}^{(k)}\right)$ represent the first and second derivatives of the regularisation functional.

\section{IMPLEMENTATION}

In order to conduct the reconstruction procedure of equation 8 we require an implementation of the forward model, and its derivatives, with respect to the optical parameters. In this work we will employ a finite element implementation of the forward model, and take the derivatives of the discretised form. 


\subsection{Finite element implementation}

\subsubsection{Forward model}

We solve the correlation diffusion equation by the finite element method. ${ }^{21-23}$ Equations 1 and 3 are each multiplied by a test function which obeys the boundary conditions, and has zeroth and first derivatives which are integrable over the domain. The boundary conditions of equation 4 are incorporated by subsequent integration by parts. The domain is subdivided into a mesh of non-overlapping elements joined at $N$ vertex nodes. On this mesh we define a set of piecewise linear basis functions such that $u_{i}\left(\mathbf{r}_{j}\right)=\delta_{i j}$ for $i, j=1, \ldots, N$ where $\mathbf{r}_{j}$ is located at the $j^{\text {th }}$ vertex node. We subsequently approximate the solution $\phi_{x}(\mathbf{r}) \approx \sum_{j}^{N} u_{j}(\mathbf{r}) \phi_{x, j}$. Selecting the basis functions in the weak formulation to be the same as the mesh basis allows us to write the resulting linear system of equations:

$$
\mathbf{A} \phi_{x}=\mathbf{q}_{x}
$$

where $\phi_{x}(\mathbf{r})$ is taken as $\phi_{a}(\mathbf{r})$ or $\phi_{0}(\mathbf{r})$, and $q_{x}(\mathbf{r})$ is $q_{a}(\mathbf{r})$ or $q_{0}(\mathbf{r})$, respectively. A is the finite element system matrix. We express the parameters of the forward model, $\mathbf{x}$, using the same basis functions such that, for example, $\mu_{a}(\mathbf{r}) \approx \sum_{k} \mu_{a, k} u_{\mu, k}(\mathbf{r})$. Consequently, for the $n$ dimensional problem,

$$
A_{i j}=\sum_{k} \int_{\Omega}\left[\kappa_{k} u_{\kappa, k}(\mathbf{r}) \nabla u_{i}(\mathbf{r}) \cdot \nabla u_{j}(\mathbf{r})+\mu_{a, k} u_{\mu, k}(\mathbf{r}) u_{i}(\mathbf{r}) u_{j}(\mathbf{r})\right] \mathrm{d}^{n} \mathbf{r}+\frac{1}{2 A} \int_{\delta \Omega} u_{i}(\mathbf{r}) u_{j}(\mathbf{r}) \mathrm{d}^{n-1} \mathbf{r} .
$$

The isotropic source term is given by

$$
q_{0, j}=\sum_{k} q_{0, k} \int_{\Omega} u_{j}(\mathbf{r}) \mathrm{d}^{n} \mathbf{r}
$$

Representing the acousto-optic modulation efficiency $\eta_{a}(\mathbf{r})$ in the mesh basis, the UOT 'virtual source' term is given by,

$$
q_{a, j}=\sum_{m} \sum_{n} \eta_{m} \phi_{0, n} \int_{\Omega} u_{m}(\mathbf{r}) u_{n}(\mathbf{r}) u_{j}(\mathbf{r}) \mathbf{d} \Omega
$$

We make measurement(s) on the boundary by applying the measurement operator $\mathbf{D}^{T}$, such that a given measurement of the AC flux is

$$
\mathbf{y}=\mathbf{D}^{T} \phi_{a}
$$

\subsubsection{Absorption and scattering sensitivity functions}

To implement the reconstruction, we must determine the derivative of the forward model with respect to each basis coefficient of $\mu_{a}(\mathbf{r})$ and $\mu_{s}^{\prime}(\mathbf{r})$,

$$
\frac{\partial \mathbf{y}}{\partial \mu_{k}}=\mathbf{D}^{T} \frac{\partial \phi_{a}}{\partial \mu_{k}}
$$

where we take $\mu_{k}$ to be equal to either $\mu_{a, k}$ or $\mu_{s, k}^{\prime}$, and understand $\mathbf{y}$ to be associated with a given index $c$ which defines the particular optical source-detector pair and acoustic configuration employed in the forward model. To find the derivative of $\phi_{a}$ with respect to each $\mu_{k}$, we begin by differentiating equation 9 with $\phi_{x}=\phi_{a}$,

$$
\frac{\partial\left(\mathbf{A} \phi_{a}\right)}{\partial \mu_{k}}=\frac{\partial \mathbf{q}_{a}}{\partial \mu_{k}} .
$$

The derivative expressed in the first term of equation 15 is expanded,

$$
\frac{\partial\left(\mathbf{A} \phi_{a}\right)}{\partial \mu_{k}}=\mathbf{A} \frac{\partial \phi_{a}}{\partial \mu_{k}}+\frac{\partial \mathbf{A}}{\partial \mu_{k}} \phi_{a},
$$

and hence,

$$
\frac{\partial \mathbf{y}}{\partial \mu_{k}}=\mathbf{D}^{T} \mathbf{A}^{-1}\left[\frac{\partial \mathbf{q}_{a}}{\partial \mu_{k}}-\frac{\partial \mathbf{A}}{\partial \mu_{k}} \phi_{a}\right]
$$


We denote the inverse of the system matrix (the discrete form Green's function) $\mathbf{G}=\mathbf{A}^{-1}$, and find the derivative of the system matrix with respect to each parameter,

$$
\begin{gathered}
\mathbf{V}_{k}^{\mu_{a}}=\frac{\partial \mathbf{A}}{\partial \mu_{a, k}}=\int_{\Omega} u_{i}(\mathbf{r}) u_{j}(\mathbf{r}) u_{k}(\mathbf{r}) \mathbf{d} r \\
\mathbf{V}_{k}^{\mu_{s}^{\prime}}=\frac{\partial \mathbf{A}}{\partial \mu_{s, k}^{\prime}}=-\frac{1}{3 \mu_{s, k}^{\prime 2}} \int_{\Omega} u_{k}(\mathbf{r}) \nabla u_{j}(\mathbf{r}) \cdot \nabla u_{m}(\mathbf{r}) \mathbf{d} r .
\end{gathered}
$$

By the application of reciprocity, ${ }^{24}$

$$
\frac{\partial \mathbf{y}}{\partial \mu_{k}}=\mathbf{D}^{T} \mathbf{A}^{-1}\left[\frac{\partial \mathbf{q}_{a}}{\partial \mu_{k}}-\mathbf{V}_{k}^{\mu} \phi_{a}\right]=\phi^{* T}\left[\frac{\partial \mathbf{q}_{a}}{\partial \mu_{k}}-\mathbf{V}_{k}^{\mu} \phi_{a}\right]
$$

Where $\phi^{*}$ solves the adjoint equation $\mathbf{A} \phi^{*}=\mathbf{q}^{+}$for the adjoint source $\mathbf{q}^{+}$defined by our measurement operator $\mathbf{D}^{T}$. We now turn our attention to the derivative of the source term,

$$
\frac{\partial q_{a, j}}{\partial \mu_{k}}=\frac{\partial}{\partial \mu_{k}} \sum_{m} \sum_{n} \eta_{m} \phi_{0, n} \int_{\Omega} u_{m}(\mathbf{r}) u_{n}(\mathbf{r}) u_{j}(\mathbf{r}) \mathbf{d} \Omega .
$$

The only term dependent upon $\mu_{a}$ or $\mu_{s}^{\prime}$ are the optical fluence coefficients, $\phi_{0}$, thus,

$$
\frac{\partial q_{a, j}}{\partial \mu_{k}}=\sum_{m} \sum_{n} \eta_{m}\left(\frac{\partial \phi_{0}}{\partial \mu_{k}}\right)_{n} \int_{\Omega} u_{m}(\mathbf{r}) u_{n}(\mathbf{r}) u_{j}(\mathbf{r}) \mathbf{d} \Omega .
$$

To find the derivative of $\phi_{0}$ we differentiate equation 9 for $\phi_{x}=\phi_{0}$,

$$
\frac{\partial\left(\mathbf{A} \phi_{0}\right)}{\partial \mu_{k}}=\frac{\partial \mathbf{q}_{\mathbf{0}}}{\partial \mu_{k}} .
$$

Expanding and simplifying, noting that the source term is independent of absorption, and, with the exception of a small region surrounding the source position, scattering,

$$
\frac{\partial \phi_{0}}{\partial \mu_{k}}=-\mathbf{A}^{-1} \frac{\partial \mathbf{A}}{\partial \mu_{k}} \phi_{0}
$$

inserting our definition of the system matrix derivative,

$$
\frac{\partial \phi_{0}}{\partial \mu_{k}}=-\mathbf{A}^{-1} \mathbf{V}_{k}^{\mu} \phi_{0},
$$

hence,

$$
\frac{\partial q_{a, j}}{\partial \mu_{k}}=-\sum_{m} \sum_{n} \eta_{m}\left(\mathbf{A}^{-1} \mathbf{V}_{k}^{\mu} \phi_{0}\right)_{n} \int_{\Omega} u_{m}(\mathbf{r}) u_{n}(\mathbf{r}) u_{j}(\mathbf{r}) \mathbf{d} \Omega .
$$

The derivatives of the forward model are thus given by,

$$
\frac{\partial \mathbf{y}}{\partial \mu_{k}}=\phi^{* T}\left[\sum_{m} \sum_{n} \eta_{m}\left(\mathbf{A}^{-1} \mathbf{V}_{k}^{\mu} \phi_{0}\right)_{n} \int_{\Omega} u_{m}(\mathbf{r}) u_{n}(\mathbf{r}) u_{j}(\mathbf{r}) \mathbf{d} \Omega-\mathbf{V}_{k}^{\mu} \phi_{a}\right] .
$$




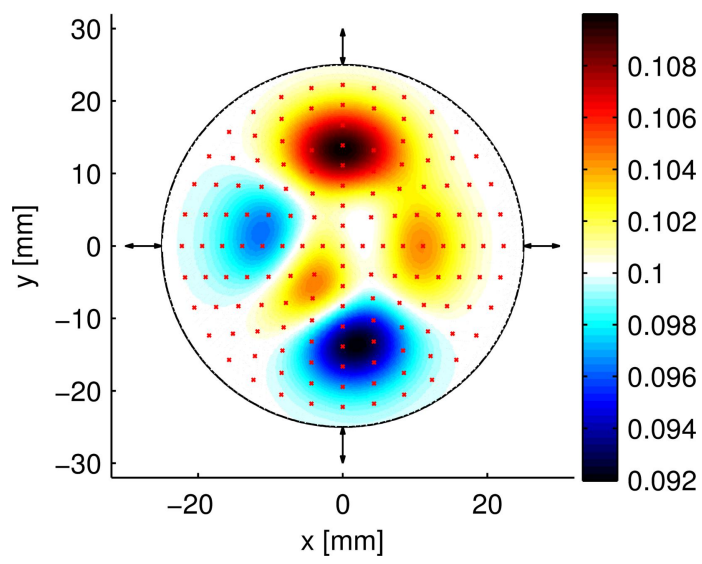

(a) Target absorption distribution $\left[\mathrm{cm}^{-1}\right]$.

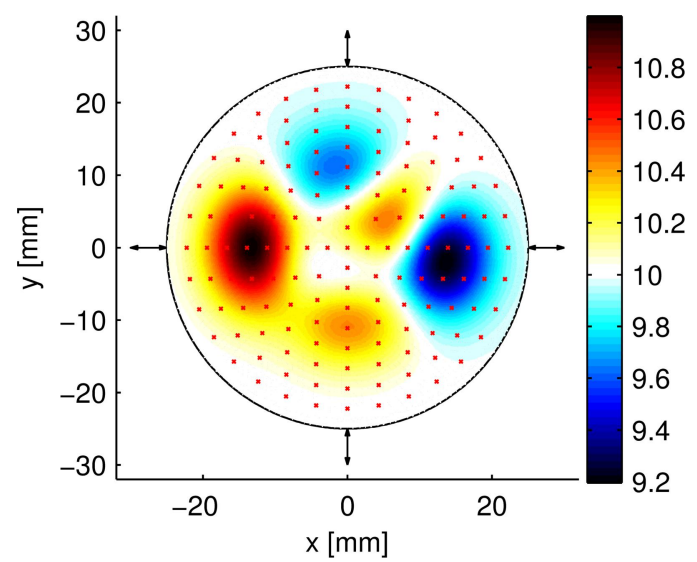

(b) Target scattering distribution $\left[\mathrm{cm}^{-1}\right]$.

Figure 1: Target absorption and scattering distributions. Co-located optical source and detector locations indicated by arrows on the boundary of the domain. The set of ultrasound scan locations is indicated by the red crosses.

\subsection{Optimisation}

In the following section we will attempt the reconstruction of a smooth distribution of $\mu_{a}$ and $\mu_{s}^{\prime}$ in a two dimensional domain. Owing to the scale of the problem tackled in this work, we directly implement the update of equation 8 as a matrix inversion using the discrete forward model and derivatives derived previously. Given the nature of the distribution we chose to implement a first-order Tikhonov regularisation scheme which penalises the gradient of the reconstructed parameters. The update is computed as

$$
\begin{aligned}
& \mathbf{x}^{(k+1)}=\mathbf{x}^{(k)}+\zeta \hat{\mathbf{H}}^{-1} \mathbf{g}, \\
& \mathbf{x}^{(k+1)}=\mathbf{x}^{(k)}+\zeta\left(\mathbf{J}^{T} \mathbf{J}+\lambda \mathbf{W}\left(\mathbf{x}^{(k)}-\mathbf{x}^{(0)}\right)\right)^{-1}\left(\mathbf{J}^{T}\left(y_{m, c}-y_{c}\left(\mathbf{x}^{(k)}\right)\right)+\lambda \mathbf{W}\right),
\end{aligned}
$$

where $\mathbf{J}$ is the block Jacobian matrix formed from the two derivatives presented in equation 27 , and the value of $\zeta$ is determined by performing a line-search along the gradient direction, $\mathrm{g}$. We perform the reconstruction on an unstructured mesh such that the discrete form of the regularisation function involves the gradient operator $\mathbf{W}$

$$
\mathbf{W}_{i j}=\int_{\Omega} \nabla u_{i}(\mathbf{r}) \nabla u_{j}(\mathbf{r}) \mathrm{d} \mathbf{r} .
$$

The use of a block Jacobian involving parameters of different scales requires that the Jacobian be appropriately scaled (pre-conditioned), lest regularisation were to overly influence one parameter over another. Such matters are considered in the context of DOT by Schweiger et al. ${ }^{20}$

\section{RESULTS}

To demonstrate the reconstruction procedure we design a target absorption and scattering distribution which we will attempt to reconstruct, as depicted in figure 1. The distributions include a number of smooth perturbations from background parameters of $\mu_{a}=0.1 \mathrm{~cm}^{-1}, \mu_{s}^{\prime}=10 \mathrm{~cm}^{-1}$, with a maximum magnitude of $\pm 10 \%$

Four collimated coherent optical sources are located normal to the boundary to which we refer by their associated index, $1:(-25,0), 2:(0,25), 3:(25,0), 4:(0,-25) \mathrm{mm}$. In simulation these are modelled by pointsources at a distance of $1 / \mu_{s}^{\prime}=0.1 \mathrm{~cm}$ below the boundary. Four diffuse detectors are co-located on the boundary (indexed as per the source positions), which integrate the diffuse light over a Gaussian profile of full-width half maximum (FWHM) of $0.1 \mathrm{~mm}$. A focused acoustic field with a Gaussian profile and FWHM of 1mm is scanned 


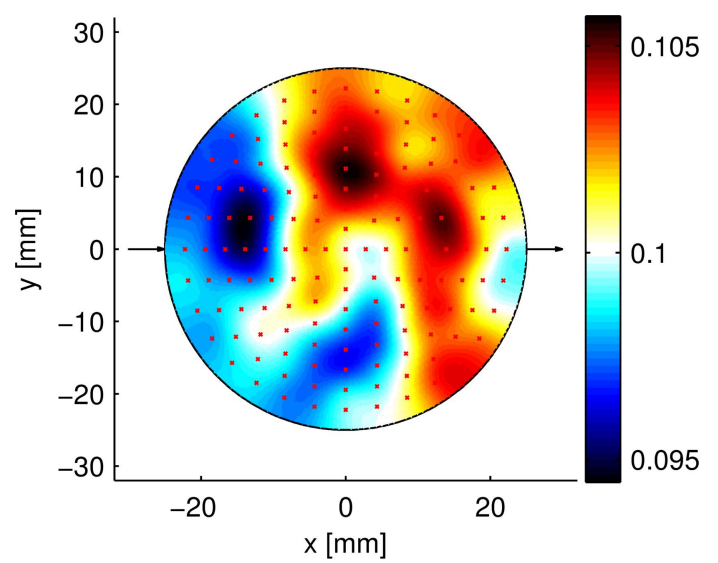

(a) Reconstructed absorption distribution $\left[\mathrm{cm}^{-1}\right]$.

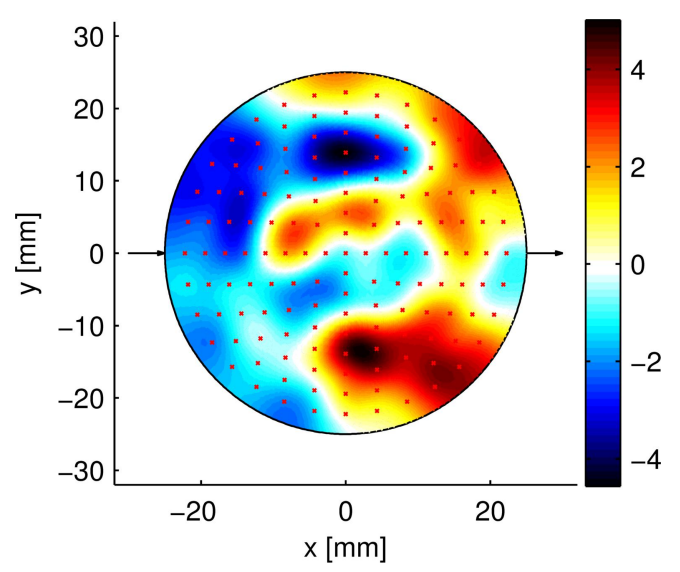

(c) Percentage error in absorption reconstruction.

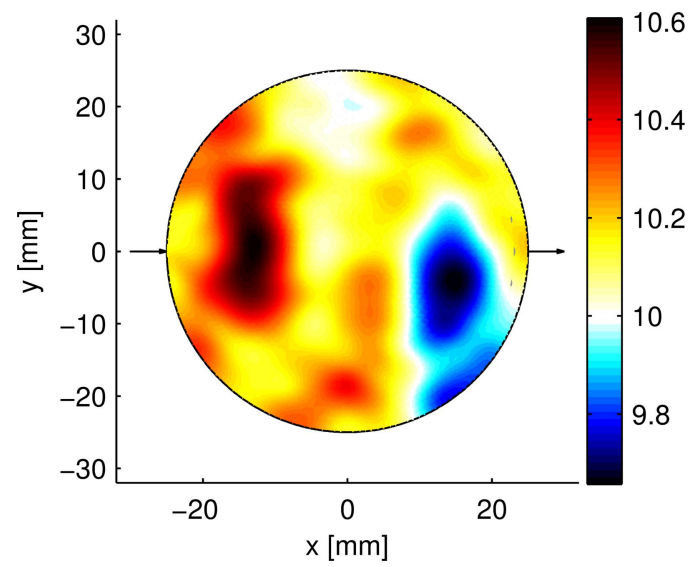

(b) Reconstructed scattering distribution $\left[\mathrm{cm}^{-1}\right]$.

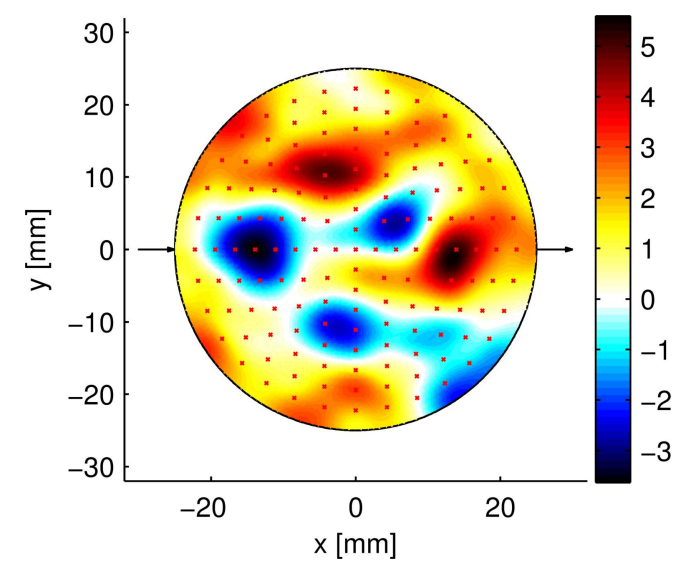

(d) Percentage error in scattering reconstruction.

Figure 2: Reconstructed parameter distributions, and percentage error of the reconstructions with respect to the target parameters, using only a single optical source-detector pair $(1,3)$.

through the plane at a series of locations indicated by the red crosses in figure 1. A simulated measurement of the diffuse outgoing modulated flux was made for each source-detector pair, under each ultrasound scan location.

In all cases, the simulated measurements are corrupted by $1 \%$ Gaussian noise, and the reconstruction procedure is initialised with values of the absorption and scattering coefficient differing by $\pm 10 \%$ respectively from the actual baseline values.

In the first reconstruction we employ optical source-detector pair $(1,3)$. Convergence was achieved after four iterations of the reconstruction algorithm, and the resultant images are shown in figure 2, alongside percentage errors with respect to the target parameters. With a limited set of internal data the reconstructed distributions vary by $\pm 5 \%$ from the target (which we recall has a maximum variation from the baseline of $\pm 10 \%$. The reconstructed absorption coefficient of figure $2 \mathrm{a}$ appears to capture the significant features of the target distribution, but the larger perturbations of the distribution (both positive and negative) are seen to be under-reported, whilst the smaller perturbations are over-reported. The scattering reconstruction of figure $2 \mathrm{~b}$ fairs less well-only the larger perturbations are discernible in the reconstructed images, the error of $5 \%$ being sufficient to completely mask their presence.

In the second reconstruction we employ all six combinations of the source-detector pairs: $(1,2),(1,3),(1,4)$, $(2,3),(2,4),(3,4)$. The simulated measurement data is again corrupted with $1 \%$ Gaussian noise, and the same 


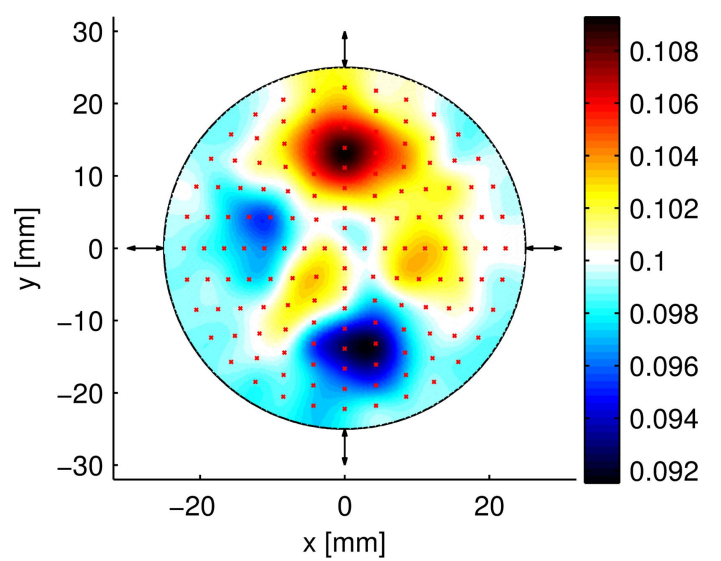

(a) Reconstructed absorption distribution $\left[\mathrm{cm}^{-1}\right]$.

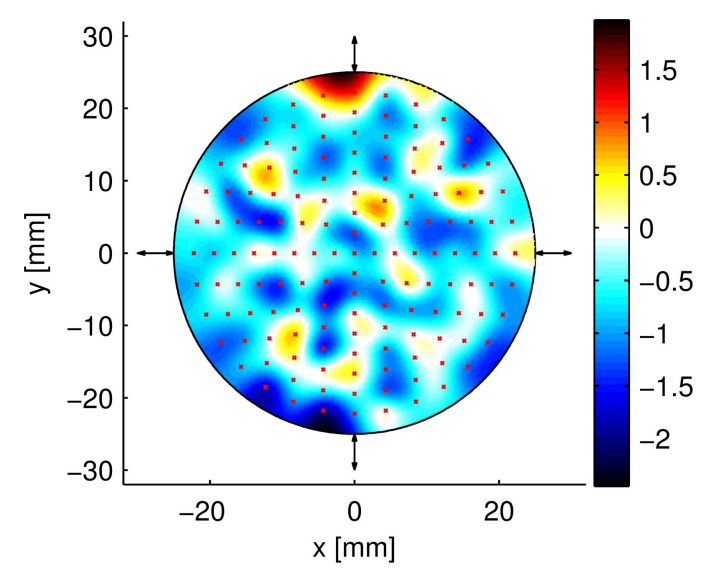

(c) Percentage error in absorption reconstruction.

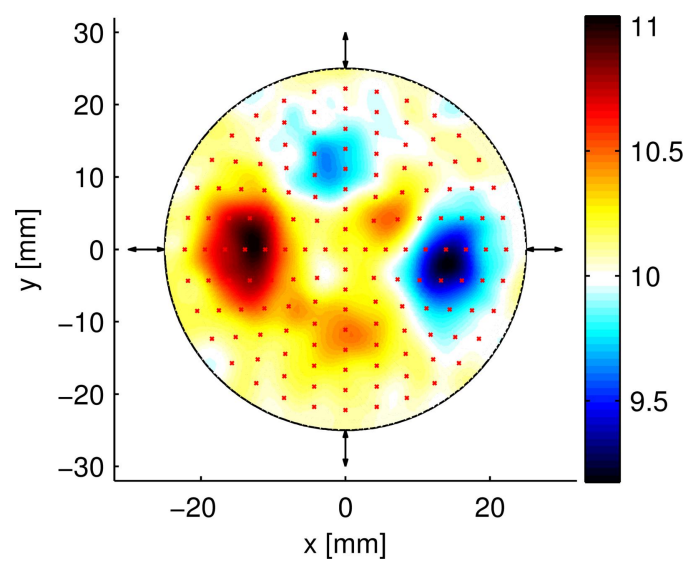

(b) Reconstructed scatttering distribution $\left[\mathrm{cm}^{-1}\right]$.

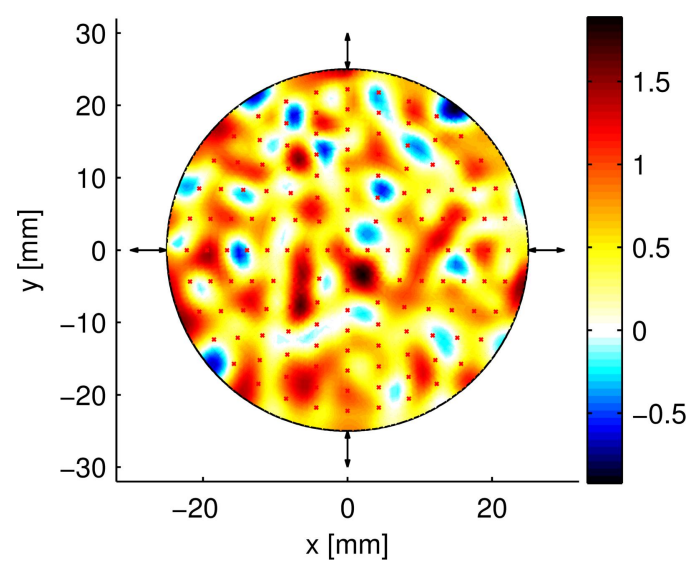

(d) Percentage error in scattering reconstruction.

Figure 3: Reconstructed parameter distributions, and percentage error of the reconstructions with respect to the target parameters, using all combinations of the source-detector pairs. 


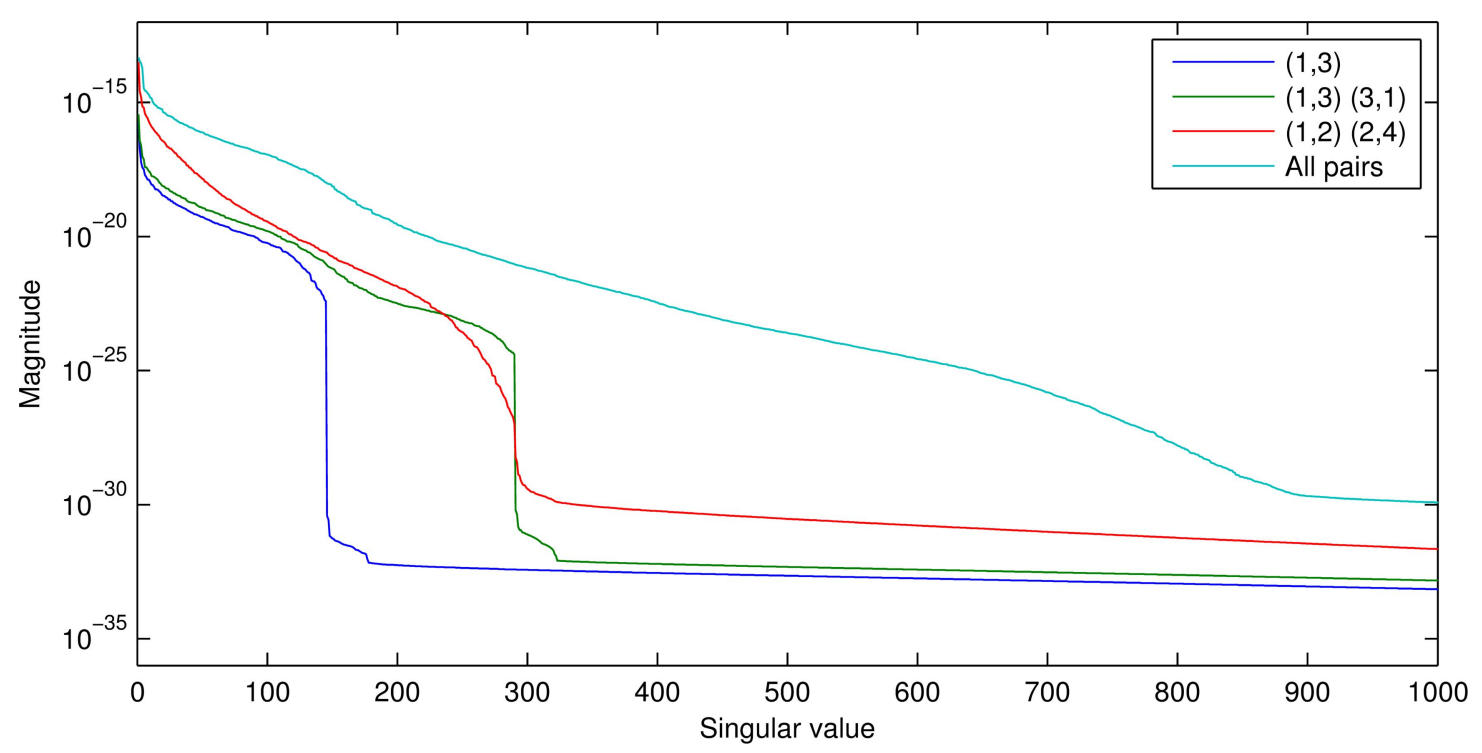

Figure 4: First 1,000 singular values of the approximation to the Hessian.

initial conditions are employed as in the case of a single source-detector pair. Convergence was again achieved after four iterations of the reconstruction algorithm, and the resultant images are shown in figure 3, alongside percentage errors with respect to the target parameters. The introduction of a significantly larger set of internal data from the alternative source-detector pairs has considerably improved the reconstruction, permitting errors of less than $+1.8 \%,-2.3 \%$ from the target. All of the features of the target absorption and scattering distribution are discernible in the reconstructed images, and the errors of figures $3 \mathrm{c}$ and $3 \mathrm{~d}$ are, by inspection, uncorrelated with the underlying parameter distribution.

The effect of introducing extra illumination profiles is seen by examining the singular value distribution of the approximation to the Hessian, $\hat{\mathbf{H}}$. Figure 4 shows that the singular values involved in the second reconstruction (that utilising all pairs of source-detectors) decay at a much slower rate than those of the first (utilising only pair $(1,3))$. Simply employing a single source-detector pair used in alternate directions $((1,3),(3,1))$ almost doubles the number of significant singular values in the spectrum. It is perhaps unsurprising that the amount of linearly independent information is increased more significantly when utilising a second source-detector pair which is spatially distinct $((1,2),(2,4))$, and in this case located orthogonally to the first pair.

\section{CONCLUSIONS}

We have derived and implemented a model-based image reconstruction procedure for UOT based around a linearised forward model. As part of this process we have developed the sensitivity functions which describe the sensitivity of the measured modulated flux to changes in the absorption and scattering distributions within a given domain.

We have explored the use of multiple optical source-detector pairs to reduce the ill-posedness of the inverse problem, and in doing so achieved a simultaneous, quantitative reconstruction of an example absorption and scattering distribution with an accuracy of $\pm 2.5 \%$ in the presence of $1 \%$ Gaussian noise.

To apply this work to real measurements will require three aspects of this work to be refined.

1. The model should be extended to three-dimensions to permit experimental realisable configurations of the optical and acoustic configuration. Whilst there are no fundamental limitations in extending the model to three-dimensions, the present implementation requires approximately $2 N$ runs of the forward model to calculate the requisite sensitivity functions, where $N$ is the number of nodes in the mesh: this may present too great a computational load for three-dimensional models. 
2. The present implementation of the reconstruction algorithm employs a straightforward matrix inversion method to update the optical parameter distribution. In three dimensions the size of the Hessian approximation $\left(\mathbf{J}^{T} \mathbf{J}\right)$ may be too large to store in memory. In this case, a matrix-free approach must be implemented.

3. A method to determine the inherent modulation efficiency, $\eta_{a}(\mathbf{r})$, must be determined. Since we expect the modulation efficiency to be proportional to the square of the acoustic pressure amplitude (over scales comparable to those used in biomedical ultrasound), it is possible that this could be determined by multiple measurements at different acoustic output pressures.

\section{ACKNOWLEDGEMENTS}

The authors would like to thank Simon Arridge, Ben Cox, Jem Hebden, Emma Malone, and Sava Sakadžić for their advice and support. This work was funded by EPSRC grant EP/G005036/1.

\section{REFERENCES}

[1] Arridge, S. R., "Methods in diffuse optical imaging.", Philosophical transactions. Series A, Mathematical, physical, and engineering sciences 369, 4558-4576 (Nov. 2011).

[2] Gross, M., Goy, P., and Al-Koussa, M., "Shot-noise detection of ultrasound-tagged photons in ultrasoundmodulated optical imaging.," Optics Letters 28, 2482-2484 (Dec. 2003).

[3] Lévêque, S., Boccara, A. C., Lebec, M., and Saint-Jalmes, H., "Ultrasonic tagging of photon paths in scattering media: parallel speckle modulation processing.," Optics Letters 24, 181-183 (Feb. 1999).

[4] Li, J., Ku, G., and Wang, L. V., "Ultrasound-modulated optical tomography of biological tissue by use of contrast of laser speckles.," Applied Optics 41, 6030-6035 (Oct. 2002).

[5] Ramaz, F., Forget, B., Atlan, M., Boccara, A. C., Gross, M., Delaye, P., and Roosen, G., "Photorefractive detection of tagged photons in ultrasound modulated optical tomography of thick biological tissues.," Optics Express 12, 5469-5474 (Nov. 2004).

[6] Murray, T. W., Sui, L., Maguluri, G., Roy, R. A., Nieva, A., Blonigen, F., and DiMarzio, C. A., "Detection of ultrasound-modulated photons in diffuse media using the photorefractive effect.," Optics Letters 29, 2509-2511 (Nov. 2004).

[7] Bossy, E., Sui, L., Murray, T. W., and Roy, R. A., "Fusion of conventional ultrasound imaging and acoustooptic sensing by use of a standard pulsed-ultrasound scanner.," Optics Letters 30, 744-746 (Apr. 2005).

[8] Li, Y., Zhang, H., Kim, C., Wagner, K. H., Hemmer, P., and Wang, L. V., "Pulsed ultrasound-modulated optical tomography using spectral-hole burning as a narrowband spectral filter.," Applied Physics Letters 93, 11111 (July 2008).

[9] Lev, A. and Sfez, B. G., "Direct, noninvasive detection of photon density in turbid media," Optics Letters 27(7), 473 (2002).

[10] Lev, A. and Sfez, B. G., "Pulsed ultrasound-modulated light tomography," Optics Letters 28(17), 1549-1551 (2003).

[11] Arridge, S. R., "Photon-measurement density functions. Part I: Analytical forms," Applied Optics 34(31), 7395-7409 (1995).

[12] Bratchenia, A., Molenaar, R., van Leeuwen, T. G., and Kooyman, R. P. H., "Acousto-optic-assisted diffuse optical tomography," Optics Letters 36(9), 1539 (2011).

[13] Powell, S. and Leung, T. S., "Linear reconstruction of absorption perturbations in coherent ultrasoundmodulated optical tomography," Journal of Biomedical Optics 18, 126020-126020 (Dec. 2013).

[14] Bal, G. and Schotland, J. C., "Inverse Scattering and Acousto-Optic Imaging," Physical Review Letters 104, 043902 (Jan. 2010).

[15] Bal, G. and Moskow, S., "Local inversions in ultrasound-modulated optical tomography," Inverse Problems 30, 025005 (Feb. 2014).

[16] Schweiger, M., Arridge, S. R., and Hiraoka, M., "The finite element method for the propagation of light in scattering media: boundary and source conditions," Medical Physics (1995). 
[17] Allmaras, M. and Bangerth, W., "Reconstructions in ultrasound modulated optical tomography," Journal of Inverse and Ill-posed Problems 19(6).

[18] Sakadžić, S. and Wang, L. V., "Correlation transfer and diffusion of ultrasound-modulated multiply scattered light," Physical Review Letters 96(16) (2006).

[19] Varma, H. M., Mohanan, K. P., Hyvönen, N., Nandakumaran, A. K., and Vasu, R. M., "Ultrasoundmodulated optical tomography: recovery of amplitude of vibration in the insonified region from boundary measurement of light correlation," Journal of the Optical Society of America A 28(11), 2322-2331 (2011).

[20] Schweiger, M., Arridge, S. R., and Nissilä, I., "Gauss-Newton method for image reconstruction in diffuse optical tomography," Physics in Medicine and Biology 50, 2365-2386 (May 2005).

[21] Arridge, S. R., "A finite element approach for modeling photon transport in tissue," Medical Physics 20(2), 299-309 (1993).

[22] Arridge, S. R. and Schweiger, M., "Photon-measurement density functions. Part 2: Finite-element-method calculations," Applied Optics 34(34), 8026-8037 (1995).

[23] Heino, J., Arridge, S., Sikora, J., and Somersalo, E., "Anisotropic effects in highly scattering media," Physical Review E 68, 031908 (Sept. 2003).

[24] Arridge, S. R., "Optical tomography in medical imaging," Inverse Problems 15, R41-R93 (Jan. 1999). 